\title{
Interactive continuing education workshops or conferences can improve professional practice and patient outcomes
}

\author{
Thomson O'Brien MA, Freemantle N, Oxman AD, et al. Continuing education meetings and workshops: effects on \\ professional practice and health care outcomes. Cochrane Database Syst Rev 2001;(2):CD003078 (latest version 9 Nov \\ 2000).

\section{QUESTION: Are continuing education meetings and workshops effective for improving professional practice and patient outcomes?}

\section{Data sources}

Studies were identified by searching the Cochrane Effective Practice and Organisation of Care Group specialised register, Medline (1966 to January 1999), the Research and Development Resource Base in Continuing Medical Education, and reference lists of relevant papers.

Sources of funding:

Alliance for Continuing

Medical Education, USA

Scottish Office Home and

Health Department, UK

Health Evidence Linkage

and Application

Network, Industry

Canada; Ministry of

Health and Social

Affairs, Norway;

Norwegian Research

Council; Society of

Medical College Director

of Continuing Medical

Education, USA; Health

Services Research Unit,

University of Aberdeen,

$U K$

For correspondence:

Ms MA Thomson

O'Brien, School of

Rehabilitation Science,

McMaster University,

Hamilton Regional

Cancer Centre,

Concession Street,

Hamilton, Ontario L8V

5C2, Canada.Fax

$+19055756308$

\section{Study selection}

Studies were selected if they were randomised controlled trials (RCTs) or quasi-experimental studies with contemporaneous data collection, involved qualified health professionals or post graduate trainees, examined the effect of planned educational activities, and objectively measured health professional practice behaviour or patient outcomes. Studies were excluded if allocation was by participant choice or if participants were all undergraduate students.

\section{Data extraction}

2 reviewers extracted data on study quality, interventions, and outcomes.

\section{Main results}

32 studies (36 comparisons, 30 RCTs) involving physicians, nurses, psychotherapists, pharmacists, and other health professionals met the selection criteria. 31 studies measured health professional behaviour and 8 measured patient outcomes. Almost all of the targeted behaviours involved learning a fairly complex set of skills. In general, studies had poor reporting of the methods used. All 32 studies compared educational meetings with a nonintervention control group; of these, 24 showed improvement in professional practice in $\geqslant 1$ major outcome among those who received the intervention. Of 8 studies that measured patient outcomes, 3 showed changes in favour of the intervention group. 1 study comparing interactive educational meetings with lectures showed no differences between groups. Of 7 RCTs with $\geqslant 1$ intervention arm comprising a didactic presentation targeting a specific behaviour, 6 showed no between group differences. Of 19 comparisons that evaluated didactic presentations combined with workshops, 11 showed moderate or moderately large intervention effects, 1 showed small effects, 3 showed no effect, and 4 had unit of analysis errors; 2 of 6 studies showed improvement in patient outcomes. 8 studies compared interactive workshops with either a non-intervention control group, a large group problem based session, or a lecture format; 7 of these studies showed improvements in professional practice in $\geqslant 1$ major outcome in the intervention groups.

\section{Conclusions}

Continuing education activities can improve professional practice and patient outcomes. Interactive workshops or conferences, alone or in combination with other interventions, are likely to be more effective than a lecture format alone.

\section{COMMENTARY}

Because of the rapid changes in health care and the emphasis on evidence based practice, nurses constantly need to update their professional knowledge and skills. In addition, regulatory organisations are increasing their expectations that professionals provide evidence of actions to maintain their professional competencies. Participation in continuing education meetings is commonly used for this purpose. This review by Thomson O'Brien $e t$ al examined the effectiveness of various continuing education delivery strategies on healthcare practice and patient outcomes. Although the review mainly reported on studies of physicians, the interventions included skills relevant for nurses.

Each study was rated for methodological rigour. Of 32 studies reviewed, protection against bias was rated as "high" in 7 studies and "moderate" in 25 studies. Despite the paucity of rigorous evaluations, the authors concluded that little support existed for the continued use of lectures alone in professional development sessions. Findings showed that interactive workshops seemed to be a more effective strategy; however, the effects of this approach were variable and should be further studied. For example, a closer examination of the effects of duration and frequency of sessions, group size, and use of follow up and practice sessions is needed. Emerging innovative uses of internet based, computer strategies to support continuing education also present an important area for future research. ${ }^{12}$ Health professionals who want to improve their practice are encouraged to choose continuing education sessions with interactive components over purely didactic interventions. Similarly, educators are advised to incorporate participant interaction in educational meetings. The findings are specific to educational interactions aimed at improving practice, and results should not be generalised to interventions for other purposes, such as professional socialisation or team building.

Ruta Valaitis, RN, MHSc

Assistant Professor, School of Nursing

McMaster University

Clinical Consultant

Hamilton Social and Public Health Services PHRED Program, Hamilton, Ontario, Canada

1 Dawes BS. Can distance learning provide a twenty-first century hallmark? AORNJ 1998;68:170-2, 174.

2 Sorensen AA. Promoting public health through electronic media: a challenge for schools of public health. Am J Public Health 2001;91:1183-6. 